\title{
Very slow convergence rates in a semilinear parabolic equation
}

\author{
Christian Stinner
}

\begin{abstract}
We consider the behavior of solutions to the Cauchy problem for a semilinear parabolic equation with supercritical nonlinearity. It is known that any slow algebraic rate appears as rate of convergence to regular steady states for some solutions. Now we show that this convergence takes place with arbitrarily slow rates which are slower than any algebraic rate, if the initial data are chosen suitably.
\end{abstract}

Mathematics Subject Classification (2000). Primary 35B40;

Secondary 35K15, 35K57.

Keywords. Convergence rate, Semilinear parabolic equation, Stationary solution.

\section{Introduction and main result}

We consider the Cauchy problem

$$
\begin{cases}u_{t}=\Delta u+|u|^{p-1} u, & x \in \mathbb{R}^{N}, t \in(0, \infty), \\ u(x, 0)=u_{0}(x), & x \in \mathbb{R}^{N},\end{cases}
$$

where $u=u(x, t), p>1, \Delta$ denotes the Laplacian operator with respect to $x$ and the function $u_{0}$ is continuous in $\mathbb{R}^{N}$ and decays to zero as $|x| \rightarrow \infty$.

After the famous work [13], this problem has been widely studied by several authors. A broad overview can be found in the monograph [24] and the references given therein. In particular, different kinds of large time behavior of classical solutions have been obtained. We refer the reader to $[5,10,16,21$, 25,27 for studies of solutions converging to zero at different algebraic rates. Moreover, the existence of growing up global solutions and different algebraic grow-up rates has been established in $[3,4,8,20,22]$. Further results concerning the large time behavior of solutions to (1.1) can be found, e.g. in $[7,11,12,18]$.

Recently, the existence of grow-up rates and rates of convergence to zero which are slower than any algebraic rate has been established in $[6,26]$. It is the 
goal of the present paper to prove a similar result for convergence to regular steady states.

With respect to existence of positive classical steady states of (1.1), the Sobolev exponent

$$
p_{s}:=\left\{\begin{array}{cl}
\infty & \text { for } N \leq 2, \\
\frac{N+2}{N-2} & \text { for } N \geq 3,
\end{array}\right.
$$

is known to be critical as such steady states only exist for $p \geq p_{s}$ (see $[1,2,14]$ ). Furthermore, there is a second critical exponent

$$
p_{c}:=\left\{\begin{array}{cl}
\infty & \text { for } N \leq 10 \\
\frac{(N-2)^{2}-4 N+8 \sqrt{N-1}}{(N-2)(N-10)} & \text { for } N \geq 11
\end{array}\right.
$$

with respect to regular steady states which satisfies $p_{c}>p_{s}$ for $N \geq 11$. More precisely, in case of $p>p_{s}$ there is a family of positive radial steady states $\varphi_{\alpha}$, $\alpha>0$, satisfying

$$
\left\{\begin{array}{l}
\varphi_{\alpha, r r}+\frac{N-1}{r} \varphi_{\alpha, r}+\varphi_{\alpha}^{p}=0, \quad r>0, \\
\varphi_{\alpha}(0)=\alpha, \quad \varphi_{\alpha, r}(0)=0,
\end{array}\right.
$$

where $r:=|x|$. Defining $\varphi_{-\alpha}(r):=-\varphi_{\alpha}(r)$ and $\varphi_{0}(r) \equiv 0$, the set $\left\{\varphi_{\alpha} \mid \alpha \in \mathbb{R}\right\}$ is a continuum of radial steady states. Moreover, for $p_{s} \leq p<p_{c}$ each pair of positive steady states of (1.1) intersects each other. But for $p \geq p_{c}$ these steady states are strictly ordered such that $\varphi_{\alpha}(|x|)$ is strictly increasing in $\alpha$ for any $x \in \mathbb{R}^{N}$ (see [27]) and

$$
\lim _{\alpha \rightarrow 0} \varphi_{\alpha}(|x|)=0, x \in \mathbb{R}^{N}, \quad \text { and } \quad \lim _{\alpha \rightarrow \infty} \varphi_{\alpha}(|x|)=\varphi_{\infty}(|x|), x \in \mathbb{R}^{N} \backslash\{0\},
$$

where $\varphi_{\infty}=\varphi_{\infty}(|x|)$ denotes the singular steady state of (1.1) which is given by

$$
\varphi_{\infty}(|x|):=L|x|^{-m}, \quad|x|>0
$$

with

$$
m:=\frac{2}{p-1} \quad \text { and } \quad L:=\{m(N-2-m)\}^{\frac{1}{p-1}} .
$$

Additionally, for $p>p_{c}$ we have

$$
\varphi_{\alpha}(|x|)=L|x|^{-m}-a_{\alpha}|x|^{-m-\lambda_{1}}+o\left(|x|^{-m-\lambda_{1}}\right), \quad|x| \simeq \infty,
$$

for any $\alpha>0$, where $a_{\alpha}>0$ is monotone decreasing in $\alpha$ (see [15]) and the constant

$$
\lambda_{1}=\lambda_{1}(N, p):=\frac{N-2-2 m-\sqrt{(N-2-2 m)^{2}-8(N-2-m)}}{2},
$$

is the smaller positive root of

$$
\lambda^{2}-(N-2-2 m) \lambda+2(N-2-m)=0 .
$$

It was shown that the steady states $\varphi_{\alpha}, \alpha>0$, are unstable for $p_{s}<p<p_{c}$, while stability results were established in case of $p \geq p_{c}$ (see $\left.[15,16,22,23]\right)$. 
Furthermore, the rates of convergence to steady states for $p>p_{c}$ were studied in $[9,17]$. There it was shown that if

$$
\begin{aligned}
& \left|u_{0}(x)\right| \leq \varphi_{\infty}(|x|), x \in \mathbb{R}^{N} \backslash\{0\}, \quad \text { and } \\
& \left|u_{0}(x)-\varphi_{\alpha}(|x|)\right| \leq c(1+|x|)^{-l}, x \in \mathbb{R}^{N},
\end{aligned}
$$

hold with some $c>0, \alpha \in \mathbb{R}$ and $l \in\left(m+\lambda_{1}, m+\lambda_{2}+2\right)$, where $\lambda_{2}$ denotes the larger positive root of (1.6), then

$$
\left\|u(\cdot, t)-\varphi_{\alpha}(|\cdot|)\right\|_{L^{\infty}\left(\mathbb{R}^{N}\right)} \leq C(1+t)^{-\frac{l-m-\lambda_{1}}{2}}, \quad t \geq 0,
$$

is satisfied and this estimate is optimal for $\alpha \neq 0$. Moreover, it was proved in [17] that this estimate cannot be extended to larger values of $l$.

In this paper we prove that there also exist rates of convergence to regular steady states which are slower than any algebraic rate. This rate will be determined by a function $\omega$ that is supposed to decrease slowly near infinity like for example $\omega(z)=\left(\ln \left(z+z_{0}\right)\right)^{-n}$ for $n>0$ or $\omega(z)=[\ln (\ln (\ldots$ $\left.\left.\left.\left(\ln \left(z+z_{0}\right)\right) \ldots\right)\right)\right]^{-1}$. Actually, the conditions on $\omega$ which are raised below are satisfied for any of these examples if $z_{0}$ is chosen large enough.

Throughout this paper, $\omega \in C^{2}([0, \infty))$ is supposed to fulfill

$$
\omega(z)>0, \quad \omega^{\prime}(z)<0 \quad \text { and } \quad \omega^{\prime \prime}(z) \geq 0 \quad \text { for all } z \geq 0
$$

such that $\omega$ satisfies

$$
\left|\frac{z \omega^{\prime}(z)}{\omega(z)}\right| \leq \tilde{c} \quad \text { and } \quad\left|\frac{z \omega^{\prime \prime}(z)}{\omega^{\prime}(z)}\right| \leq C_{\omega} \quad \text { for all } z \geq 0
$$

with some constants $\tilde{c}>0$ and

$$
C_{\omega} \in\left(0, N-1-2\left(m+\lambda_{1}\right)\right) .
$$

We remark that (1.9) seems to be necessary in order to estimate terms involving derivatives of $\omega$ (see Lemmas 3.1 and 4.2). As $N-1-2\left(m+\lambda_{1}\right)>1$ holds in case of $p>p_{c}$, it is possible to choose $C_{\omega}>1$ and, as can easily be checked, $\omega$ can be chosen to be a power of a logarithm or an iterated logarithm as mentioned above.

Now we state our main result.

Theorem 1.1. Suppose $N \geq 11$ and $p>p_{c}$. Moreover, let $u_{0} \in C^{0}\left(\mathbb{R}^{N}\right)$ satisfy

$$
\left|u_{0}(x)\right|<\varphi_{\infty}(|x|), \quad x \in \mathbb{R}^{N} \backslash\{0\},
$$

and let $\omega \in C^{2}([0, \infty))$ meet the conditions $(1.7)-(1.9)$.

(a) If $u_{0}$ satisfies (1.10) and

$$
\left|u_{0}(x)-\varphi_{\alpha}(|x|)\right| \leq c_{1}(1+|x|)^{-m-\lambda_{1}} \omega(|x|), \quad x \in \mathbb{R}^{N},
$$

with some constants $\alpha \in \mathbb{R}$ and $c_{1}>0$, then there are positive constants $B_{1}$ and $C_{1}$ such that the solution $u$ of (1.1) fulfills

$$
\left\|u(\cdot, t)-\varphi_{\alpha}(|\cdot|)\right\|_{L^{\infty}\left(\mathbb{R}^{N}\right)} \leq C_{1} \omega\left(B_{1}(t+1)^{\frac{1}{2}}\right) \quad \text { for all } t \geq 0 .
$$


(b) Estimate (1.12) is optimal for any $\alpha \neq 0$ in the following sense. Given $\alpha \neq 0$, if $u_{0}$ fulfills (1.10) and

$\begin{cases}u_{0}(x)-\varphi_{\alpha}(|x|) \geq c_{2}(1+|x|)^{-m-\lambda_{1}} \omega(|x|), x \in \mathbb{R}^{N}, & \text { if } \alpha>0, \\ u_{0}(x)-\varphi_{\alpha}(|x|) \leq-c_{2}(1+|x|)^{-m-\lambda_{1}} \omega(|x|), x \in \mathbb{R}^{N}, & \text { if } \alpha<0,\end{cases}$

with some $c_{2}>0$, then

$$
\left\|u(\cdot, t)-\varphi_{\alpha}(|\cdot|)\right\|_{L^{\infty}\left(\mathbb{R}^{N}\right)} \geq C_{2} \omega\left(B_{2}(t+1)^{\frac{1}{2}}\right) \quad \text { for all } t \geq 0
$$

holds for the solution $u$ of (1.1) with some positive constants $B_{2}$ and $C_{2}$.

Observe that, in particular, (1.12) and (1.13) indicate that arbitrarily slow rates of stabilization towards regular equilibria occur in each problem (1.1) when $p>p_{c}$. To the best of our knowledge, such a behavior has not yet been detected in the context of parabolic equations, the only exception being the recent work [26], where a similar result has been established for the rates at which certain solutions of (1.1) decay to zero as $t \rightarrow \infty$. Although our approach is mainly based on the ideas of [9], we need some additional ingredients to obtain these very slow convergence rates. Similar ideas can be found in the recent proofs of very slow grow-up rates and rates of convergence to zero in $[6,26]$, but our concept is simpler as we can use a linearized problem instead of the nonlinear problem.

We remark that a proof of similar results in the critical case $p=p_{c}$ would require different ideas. This is essentially due to the fact that in the asymptotic behavior of the corresponding steady states a logarithmic factor appears according to

$$
\varphi_{\alpha}(|x|)=L|x|^{-m}-a_{\alpha}|x|^{-m-\lambda_{1}} \log (|x|)+o\left(|x|^{-m-\lambda_{1}} \log (|x|)\right), \quad|x| \simeq \infty,
$$

for $\alpha>0$ and $p=p_{c}$, where $a_{\alpha}$ is positive (see $[15,19]$ ).

This paper is structured in the following way. In Sect. 2 we recall some results of [9] concerning certain linearized problems. In Sects. 3 and 4 we establish a suitable upper and lower bound for solutions of these linearized problems, respectively. These estimates are used to prove two results which are slightly more general than Theorem 1.1 and estimate the evolution of the difference of two solutions of (1.1) (see Propositions 3.4 and 4.4). Finally, the two parts of Theorem 1.1 are proved at the end of Sects. 3 and 4, respectively.

\section{Preliminaries}

In order to prove our main result, the procedures in $[9,17]$ suggest to study the linearization of (1.1) around its steady states $\varphi_{\alpha}$. In this section, we recall some results of [9] that will be used in the forthcoming sections. Hence, for $\alpha>0$ we define the linear operator

$$
P_{\alpha} U:=U_{r r}+\frac{N-1}{r} U_{r}+p \varphi_{\alpha}^{p-1} U
$$


and consider solutions $U=U(r, t)$ of the problem

$$
\begin{cases}U_{t}=P_{\alpha} U, & r>0, t>0, \\ U_{r}(0, t)=0, & t>0, \\ U(r, 0)=U_{0}(r), & r \geq 0,\end{cases}
$$

where $U_{0}$ is a continuous function decaying to zero as $r \rightarrow \infty$. Furthermore, let $\psi(r)$ satisfy

$$
\left\{\begin{array}{l}
P_{\alpha} \psi=0, \quad r>0, \\
\psi(0)=1, \quad \psi_{r}(0)=0
\end{array}\right.
$$

and let $\Psi(r)$ denote the solution to

$$
\begin{cases}P_{\alpha} \Psi=\psi, & r>0, \\ \Psi(0)=0, & \Psi_{r}(0)=0 .\end{cases}
$$

Next we state some useful properties of $\psi$ and $\Psi$, which were proved in Lemmas 2.3 and 2.4 of [9].

Lemma 2.1. Let $N \geq 11, p>p_{c}$ and $\alpha>0$. Then the solution $\psi$ of $(2.2)$ is given by $\psi(r):=\frac{\partial}{\partial \alpha} \varphi_{\alpha}(r), r \geq 0$. Moreover, $\psi$ and the solution $\Psi$ of $(2.3)$ have the following properties:

(i) $\psi(r)$ is positive for $r \geq 0$ and satisfies

$$
\psi(r)=c_{\alpha} r^{-m-\lambda_{1}}+o\left(r^{-m-\lambda_{1}}\right) \quad \text { as } r \rightarrow \infty,
$$

where $c_{\alpha}$ is a positive constant.

(ii) $\Psi(r)$ is positive for all $r>0$ and fulfills

$$
\Psi(r)=C_{\alpha} r^{-m-\lambda_{1}+2}+o\left(r^{-m-\lambda_{1}+2}\right) \quad \text { as } r \rightarrow \infty
$$

with a positive constant $C_{\alpha}$.

Finally we recall two comparison results of [9]. Therefore, let $u$ and $\tilde{u}$ be solutions of (1.1) evolving from initial data $u_{0}$ and $\tilde{u}_{0}$, respectively. The results of [9] indicate that it is sufficient to estimate the solution $U$ of (2.1) in order to obtain the bounds for $u-\tilde{u}$ which are stated in our main result (with $\left.\tilde{u}=\varphi_{\alpha}\right)$.

Lemma 2.2. Suppose $N \geq 11$ and $p>p_{c}$.

(i) If $u_{0}, \tilde{u}_{0}$ and $U_{0}$ satisfy

$\left|u_{0}(x)\right|,\left|\tilde{u}_{0}(x)\right| \leq \varphi_{\alpha}(|x|) \quad$ and $\quad U_{0}(|x|) \geq\left|u_{0}(x)-\tilde{u}_{0}(x)\right|, \quad x \in \mathbb{R}^{N}$, with some $\alpha>0$, then the solutions $U$ of (2.1) and $u, \tilde{u}$ of (1.1) fulfill

$$
U(|x|, t) \geq|u(x, t)-\tilde{u}(x, t)|, \quad \text { for all } x \in \mathbb{R}^{N}, t>0 .
$$

(ii) If $u_{0}, \tilde{u}_{0}$ and $U_{0}$ fulfill

$$
\begin{aligned}
\varphi_{\alpha}(|x|) & \leq \tilde{u}_{0}(x) \leq u_{0}(x) \leq \varphi_{\infty}(|x|), \quad x \in \mathbb{R}^{N} \backslash\{0\}, \quad \text { and } \\
0 & \leq U_{0}(|x|) \leq u_{0}(x)-\tilde{u}_{0}(x), \quad x \in \mathbb{R}^{N},
\end{aligned}
$$

with some $\alpha>0$, then the solutions $U$ of (2.1) and $u, \tilde{u}$ of (1.1) satisfy

$$
0 \leq U(|x|, t) \leq u(x, t)-\tilde{u}(x, t), \quad \text { for all } x \in \mathbb{R}^{N}, t>0 .
$$




\section{Upper bound}

In this section we prove the upper bound of the very slow convergence rate for the solutions of (1.1) which is claimed in Theorem 1.1(a). Therefore, we construct suitable supersolutions of (2.1). We use the ideas presented in [9] and this section is similar to Sect. 3 in [9]. But in order to handle terms involving $\omega$, some additional ideas are necessary.

We first construct a supersolution $U_{\text {out }}$ of (2.1) which decays in an appropriate way as $r \rightarrow \infty$. Here, the condition (1.9) seems to be necessary in order to control terms involving $\omega^{\prime \prime}$.

Lemma 3.1. Let $N \geq 11, p>p_{c}$ and $\alpha>0$. Then

$$
U_{\text {out }}(r):=r^{-m-\lambda_{1}} \omega(r), \quad r>0,
$$

fulfills $P_{\alpha} U_{\text {out }} \leq 0$.

Proof. As the steady states $\varphi_{\alpha}$ are ordered, we obtain for $r>0$ due to (1.7), (1.8), and the fact that $\left(m+\lambda_{1}\right)^{2}-(N-2)\left(m+\lambda_{1}\right)+p L^{p-1}=0$ by (1.6)

$$
\begin{aligned}
P_{\alpha} U_{\text {out }}= & U_{\text {out }, r r}+\frac{N-1}{r} U_{\text {out }, r}+p \varphi_{\alpha}^{p-1} U_{\text {out }} \\
\leq & U_{\text {out }, r r}+\frac{N-1}{r} U_{\text {out }, r}+p \varphi_{\infty}^{p-1} U_{\text {out }} \\
= & \left(m+\lambda_{1}\right)\left(m+\lambda_{1}+1\right) r^{-m-\lambda_{1}-2} \omega(r)-2\left(m+\lambda_{1}\right) r^{-m-\lambda_{1}-1} \omega^{\prime}(r) \\
& +r^{-m-\lambda_{1}} \omega^{\prime \prime}(r)-\frac{N-1}{r}\left(m+\lambda_{1}\right) r^{-m-\lambda_{1}-1} \omega(r) \\
& +\frac{N-1}{r} r^{-m-\lambda_{1}} \omega^{\prime}(r)+p \frac{L^{p-1}}{r^{m(p-1)}} r^{-m-\lambda_{1}} \omega(r) \\
= & {\left[\left(m+\lambda_{1}\right)^{2}-(N-2)\left(m+\lambda_{1}\right)+p L^{p-1}\right] r^{-m-\lambda_{1}-2} \omega(r) } \\
& +\left[N-1-2\left(m+\lambda_{1}\right)\right] r^{-m-\lambda_{1}-1} \omega^{\prime}(r)+r^{-m-\lambda_{1}} \omega^{\prime \prime}(r) \\
= & -\left[\left(N-1-2\left(m+\lambda_{1}\right)\right)\left|\omega^{\prime}(r)\right|-r \omega^{\prime \prime}(r)\right] r^{-m-\lambda_{1}-1} \\
\leq & -\left[\left(N-1-2\left(m+\lambda_{1}\right)\right)-C_{\omega}\right] r^{-m-\lambda_{1}-1}\left|\omega^{\prime}(r)\right| .
\end{aligned}
$$

Now the claim is a consequence of (1.9).

Next we construct a supersolution $U_{\text {in }}$ of (2.1) which decays at $r=0$ with the rate claimed in Theorem 1.1. Our choice of $U_{\text {in }}$ is a generalization of the function used in [9]. In particular, we modify the argument of $\omega$ in order to obtain the desired results.

Lemma 3.2. Suppose $N \geq 11, p>p_{c}$ and $\alpha>0$. Then there are positive constants $B, c$ and $\tau_{0} \geq 1$ such that for any $\tau \geq \tau_{0}$ the function

$U_{\text {in }}(r, t):=\omega\left(B(t+\tau)^{\frac{1}{2}}\right) \psi(r)+\frac{B}{2}(t+\tau)^{-\frac{1}{2}} \omega^{\prime}\left(B(t+\tau)^{\frac{1}{2}}\right) \Psi(r), \quad r>0, t>0$, satisfies

(i) $U_{\text {in }, t} \geq P_{\alpha} U_{\text {in }}$ for all $r>0$ and $t>0$,

(ii) $U_{\text {in }}(r, t)>0$ for $t \geq 0$ and $r \in\left[0, B(t+\tau)^{\frac{1}{2}}\right]$,

(iii) $U_{\text {in }}(r, t)>c U_{\text {out }}(r)$ for $t \geq 0$ and $r=B(t+\tau)^{\frac{1}{2}}$. 
Proof. For every $B>0$ and $\tau>0,(2.2)$ and (2.3) imply (omitting the argument $B(t+\tau)^{\frac{1}{2}}$ of $\omega, \omega^{\prime}$ and $\left.\omega^{\prime \prime}\right)$

$$
\begin{aligned}
U_{\text {in }, t}-P_{\alpha} U_{\text {in }}= & \frac{B}{2}(t+\tau)^{-\frac{1}{2}} \omega^{\prime} \psi(r)+\frac{B^{2}}{4}(t+\tau)^{-1} \omega^{\prime \prime} \Psi(r) \\
& -\frac{B}{4}(t+\tau)^{-\frac{3}{2}} \omega^{\prime} \Psi(r)-\omega P_{\alpha} \psi(r)-\frac{B}{2}(t+\tau)^{-\frac{1}{2}} \omega^{\prime} P_{\alpha} \Psi(r) \\
= & \frac{B^{2}}{4}(t+\tau)^{-1} \omega^{\prime \prime} \Psi(r)-\frac{B}{4}(t+\tau)^{-\frac{3}{2}} \omega^{\prime} \Psi(r) \text { for } r>0, t>0 .
\end{aligned}
$$

Thus, (1.7) implies (i) as $\Psi$ is nonnegative by Lemma 2.1.

To prove (ii) and (iii) we proceed like in [9] and fix some constants. By Lemma 2.1 there are positive constants $c_{\alpha}^{-}$and $C_{\alpha}^{+}$such that

$$
\psi(r) \geq c_{\alpha}^{-} r^{-m-\lambda_{1}} \quad \text { and } \quad \Psi(r) \leq C_{\alpha}^{+} r^{-m-\lambda_{1}+2} \quad \text { for } r \geq 1
$$

hold. Next we fix $B>0$ such that

$$
c_{\alpha}^{-}-\frac{B^{2}}{2} \tilde{c} C_{\alpha}^{+}>0
$$

is fulfilled, where $\tilde{c}$ is given by (1.8). Then we choose $c \in\left(0, c_{\alpha}^{-}-\frac{B^{2}}{2} \tilde{c} C_{\alpha}^{+}\right)$. Finally, by Lemma 2.1 we are able to choose $\tau_{0} \geq 1$ such that $B \tau_{0}^{\frac{1}{2}} \geq 1$ and

$$
\psi(r)-\frac{\tilde{c}}{2 \tau_{0}} \Psi(r)>0 \quad \text { for all } r \in[0,1] .
$$

Now we fix some $\tau \geq \tau_{0}$. Then for $r \in[0,1]$ and $t \geq 0$, due to Lemma 2.1, (1.8) and the choice of $\tau$ we obtain

$$
\begin{aligned}
U_{\text {in }}(r, t) & \geq \omega\left(B(t+\tau)^{\frac{1}{2}}\right)\left[\psi(r)-\frac{B}{2}(t+\tau)^{-\frac{1}{2}} \frac{\tilde{c}}{B(t+\tau)^{\frac{1}{2}}} \Psi(r)\right] \\
& \geq \omega\left(B(t+\tau)^{\frac{1}{2}}\right)\left[\psi(r)-\frac{\tilde{c}}{2 \tau} \Psi(r)\right]>0 .
\end{aligned}
$$

Moreover, for $t \geq 0$ and $r \in\left[1, B(t+\tau)^{\frac{1}{2}}\right]$, by Lemma 2.1, (1.8), (3.1), (3.2) and $B \tau^{\frac{1}{2}} \geq 1$ we conclude

$$
\begin{aligned}
U_{\text {in }}(r, t) & \geq \omega\left(B(t+\tau)^{\frac{1}{2}}\right)\left[\psi(r)-\frac{B}{2}(t+\tau)^{-\frac{1}{2}} \frac{\tilde{c}}{B(t+\tau)^{\frac{1}{2}}} \Psi(r)\right] \\
& \geq \omega\left(B(t+\tau)^{\frac{1}{2}}\right)\left[c_{\alpha}^{-} r^{-m-\lambda_{1}}-\frac{\tilde{c}}{2(t+\tau)} C_{\alpha}^{+} r^{-m-\lambda_{1}+2}\right] \\
& \geq \omega\left(B(t+\tau)^{\frac{1}{2}}\right) r^{-m-\lambda_{1}}\left[c_{\alpha}^{-}-\frac{B^{2}}{2} \tilde{c} C_{\alpha}^{+}\right]>0 .
\end{aligned}
$$

Hence, we have proved (ii). In particular, in case of $t \geq 0$ and $r=B(t+\tau)^{\frac{1}{2}}$, (3.3) implies

$$
U_{\text {in }}(r, t) \geq \omega\left(B(t+\tau)^{\frac{1}{2}}\right) r^{-m-\lambda_{1}}\left[c_{\alpha}^{-}-\frac{B^{2}}{2} \tilde{c} C_{\alpha}^{+}\right]>c r^{-m-\lambda_{1}} \omega(r)
$$

due to the choice of $c$. Thus, (iii) is shown and the claim is proved. 
Now we use the supersolutions $U_{\text {in }}$ and $U_{\text {out }}$ to obtain a suitable estimate from above for the solution of (2.1). The proof is similar to the one presented in Proposition 3.3 of [9], but we include a short proof for the sake of completeness.

Lemma 3.3. Assume $N \geq 11, p>p_{c}$ and $U_{0} \in C^{0}([0, \infty))$ such that

$$
0<U_{0}(r) \leq(1+r)^{-m-\lambda_{1}} \omega(r) \quad \text { for } r \geq 0 .
$$

Then the solution $U$ of (2.1) fulfills

$$
\|U(\cdot, t)\|_{L^{\infty}((0, \infty))} \leq C \omega\left(B(t+1)^{\frac{1}{2}}\right) \quad \text { for all } t \geq 0
$$

with some positive constants $B$ and $C$.

Proof. We use the constants $B, c$ and $\tau_{0}$ given in Lemma 3.2 and fix some $\tau \geq \tau_{0}$. Moreover, let $U_{\text {out }}$ and $U_{\text {in }}$ denote the functions defined in Lemmas 3.1 and 3.2, respectively. By Lemma 3.2, the function

$$
r^{*}(t):=\sup \left\{r>0 \mid U_{\text {in }}(\rho, t)<c U_{\text {out }}(\rho) \text { for all } \rho \in[0, r)\right\}
$$

is well-defined and satisfies $r^{*}(t) \in\left(0, B(t+\tau)^{\frac{1}{2}}\right)$ for all $t \geq 0$. Setting now

$$
U^{+}(r, t):= \begin{cases}U_{\text {in }}(r, t) & \text { for } 0 \leq r<r^{*}(t), \\ c U_{\text {out }}(r) & \text { for } r \geq r^{*}(t),\end{cases}
$$

and $t \geq 0$, there is some large constant $C_{1}>0$ such that

$$
0<U_{0}(r)<C_{1} U^{+}(r, 0) \text { for } r \geq 0
$$

is fulfilled due to Lemma 3.2 (ii). As furthermore $U_{r}^{+}(0, t)=0$ holds due to $\psi_{r}(0)=\Psi_{r}(0)=0, C_{1} U^{+}$is a supersolution of (2.1) by Lemmas 3.1 and 3.2. Hence, the comparison principle implies

$$
0<U(r, t) \leq C_{1}^{+} U^{+}(r, t) \text { for } r \geq 0 \text { and } t \geq 0 .
$$

Moreover, we conclude by Lemmas 2.1, 3.1, 3.2, (1.7) and the definition of $r^{*}(t)$

$$
\begin{aligned}
\left\|U^{+}(\cdot, t)\right\|_{L^{\infty}((0, \infty))} & \leq \max \left\{\omega\left(B(t+\tau)^{\frac{1}{2}}\right)\|\psi\|_{L^{\infty}((0, \infty))}, c\left\|U_{\text {out }}\right\|_{L^{\infty}\left(\left(r^{*}(t), \infty\right)\right)}\right\} \\
& =\max \left\{\omega\left(B(t+\tau)^{\frac{1}{2}}\right)\|\psi\|_{L^{\infty}((0, \infty))}, c U_{\text {out }}\left(r^{*}(t)\right)\right\} \\
& =\omega\left(B(t+\tau)^{\frac{1}{2}}\right)\|\psi\|_{L^{\infty}((0, \infty))} \leq C_{2} \omega\left(B(t+1)^{\frac{1}{2}}\right)
\end{aligned}
$$

for all $t \geq 0$ with some positive constant $C_{2}$.

Now we immediately obtain a generalization of Theorem 1.1(a) which describes the evolution of the difference of two solutions to (1.1).

Proposition 3.4. Suppose $N \geq 11$ and $p>p_{c}$. Moreover, let $u_{0}, \tilde{u}_{0} \in C^{0}\left(\mathbb{R}^{N}\right)$ satisfy

$$
\left|u_{0}(x)\right|,\left|\tilde{u}_{0}(x)\right| \leq \varphi_{\alpha}(|x|), \quad x \in \mathbb{R}^{N}
$$

and

$$
\left|u_{0}(x)-\tilde{u}_{0}(x)\right| \leq c_{1}(1+|x|)^{-m-\lambda_{1}} \omega(|x|), \quad x \in \mathbb{R}^{N},
$$


with some positive constants $\alpha$ and $c_{1}$. Then there are positive constants $B_{1}$ and $C_{1}$ such that the solutions $u$ and $\tilde{u}$ of (1.1) fulfill

$$
\|u(\cdot, t)-\tilde{u}(\cdot, t)\|_{L^{\infty}\left(\mathbb{R}^{N}\right)} \leq C_{1} \omega\left(B_{1}(t+1)^{\frac{1}{2}}\right) \quad \text { for all } t \geq 0 .
$$

Proof. By choosing

$$
U_{0}(r):=c_{1}(1+r)^{-m-\lambda_{1}} \omega(r), \quad r \geq 0,
$$

we obtain

$$
\left|u_{0}(x)-\tilde{u}_{0}(x)\right| \leq U_{0}(|x|), \quad x \in \mathbb{R}^{N} .
$$

Hence, Lemmas 2.2 (i) and 3.3 imply

$\|u(\cdot, t)-\tilde{u}(\cdot, t)\|_{L^{\infty}\left(\mathbb{R}^{N}\right)} \leq\|U(\cdot, t)\|_{L^{\infty}((0, \infty))} \leq C \omega\left(B(t+1)^{\frac{1}{2}}\right) \quad$ for all $t \geq 0$ with some positive constants $B$ and $C$.

Finally, the first part of Theorem 1.1 can easily be proved.

Proof of Theorem 1.1(a): (1.10) and (1.11) imply the existence of some $\tilde{\alpha}>|\alpha|$ such that

$$
\left|u_{0}(x)\right| \leq \varphi_{\tilde{\alpha}}(|x|), \quad x \in \mathbb{R}^{N}
$$

holds (due to (1.3) and (1.4)). Hence, the claim is a consequence of Proposition 3.4 with $\tilde{\alpha}$ and $\tilde{u}_{0}=\varphi_{\alpha}$.

\section{Lower bound}

In this section we prove Theorem 1.1(b). Therefore, we construct a suitable subsolution of (2.1). Again, we adapt ideas of [9] and this section corresponds to Sect. 4 in [9].

First we give a subsolution $U_{\text {in }}$ of (2.1) which decays for $r=0$ with the rate claimed in Theorem 1.1 .

Lemma 4.1. Let $N \geq 11$ and $p>p_{c}$. Then for any $B>0$ the function

$$
U_{\text {in }}(r, t):=\omega\left(B(t+1)^{\frac{1}{2}}\right) \psi(r), \quad r>0, t>0,
$$

is a subsolution of (2.1).

Proof. Fixing some $B>0$, we obtain due to (1.7), (2.2) and Lemma 2.1

$$
\begin{aligned}
U_{\text {in }, t}-P_{\alpha} U_{\text {in }} & =\frac{B}{2}(t+1)^{-\frac{1}{2}} \omega^{\prime}\left(B(t+1)^{\frac{1}{2}}\right) \psi(r)-\omega\left(B(t+1)^{\frac{1}{2}}\right) P_{\alpha} \psi(r) \\
& =\frac{B}{2}(t+1)^{-\frac{1}{2}} \omega^{\prime}\left(B(t+1)^{\frac{1}{2}}\right) \psi(r) \leq 0 \text { for } r>0, t>0 .
\end{aligned}
$$

Hence, the claim is proved.

Now we construct a subsolution $U_{\text {out }}$ of (2.1) which decays in a suitable way as $r \rightarrow \infty$. In order to obtain a subsolution with a similar structure as compared to the function used in [9], there is some additional effort to do. In particular, condition (1.9) again seems to be necessary in order to handle terms involving $\omega^{\prime \prime}$. We remark that the conditions imposed below on $k$ and $b_{2}$ 
make sense, because (1.5) implies $N-2-m-\lambda_{1}>0$ and $N-1-2\left(m+\lambda_{1}\right)>0$ and $C_{\omega}$ fulfills (1.9).

Lemma 4.2. Suppose $N \geq 11, p>p_{c}$ and let $b_{1}, b_{2}$ and $k$ be positive constants such that

$$
0<k<\min \left\{1, \frac{N-2-m-\lambda_{1}}{2}, \frac{N-1-2\left(m+\lambda_{1}\right)-C_{\omega}}{4}\right\}
$$

and

$$
b_{2} \geq\left(\frac{\left(N-2-m-\lambda_{1}\right)\left(m+\lambda_{1}\right)+\tilde{c}\left(N-1-2\left(m+\lambda_{1}\right)\right)}{k}\right)^{k} b_{1}
$$

hold, where $C_{\omega}$ satisfies (1.9) and $\tilde{c}$ is given by (1.8). Then the function

$$
\begin{aligned}
U_{\text {out }}(r, t):= & \max \left\{0, b_{1} r^{-m-\lambda_{1}} \omega(r)-b_{2}(1+t)^{k} r^{-m-\lambda_{1}-2 k} \omega(r)\right\}, \\
& r>0, t \geq 0,
\end{aligned}
$$

is a subsolution of (2.1).

Proof. As $U \equiv 0$ is a subsolution of (2.1) we restrict ourselves to the case where $U_{\text {out }}$ is positive.

Thus, we assume $r^{2}>\left(\frac{b_{2}}{b_{1}}\right)^{\frac{1}{k}}(1+t)$. Using the choices of $b_{1}, b_{2}$ and $k$, we obtain by (1.7) and (1.8)

$$
\begin{aligned}
U_{\text {out },}, & -P_{\alpha} U_{\text {out }} \\
\leq & U_{\text {out }, t}-U_{\text {out }, r r}-\frac{N-1}{r} U_{\text {out }, r} \\
= & -k b_{2}(1+t)^{k-1} r^{-m-\lambda_{1}-2 k} \omega(r) \\
& +b_{1}\left(N-2-m-\lambda_{1}\right)\left(m+\lambda_{1}\right) r^{-m-\lambda_{1}-2} \omega(r) \\
& -b_{1}\left(N-1-2\left(m+\lambda_{1}\right)\right) r^{-m-\lambda_{1}-1} \omega^{\prime}(r)-b_{1} r^{-m-\lambda_{1}} \omega^{\prime \prime}(r) \\
& -b_{2}(1+t)^{k}\left(N-2-m-\lambda_{1}-2 k\right)\left(m+\lambda_{1}+2 k\right) r^{-m-\lambda_{1}-2 k-2} \omega(r) \\
& +b_{2}(1+t)^{k}\left(N-1-2\left(m+\lambda_{1}\right)-4 k\right) r^{-m-\lambda_{1}-2 k-1} \omega^{\prime}(r) \\
& +b_{2}(1+t)^{k} r^{-m-\lambda_{1}-2 k} \omega^{\prime \prime}(r) \\
\leq & -k b_{2}(1+t)^{k-1} r^{-m-\lambda_{1}-2 k} \omega(r) \\
& +b_{1}\left[\left(N-2-m-\lambda_{1}\right)\left(m+\lambda_{1}\right)+\tilde{c}\left(N-1-2\left(m+\lambda_{1}\right)\right)\right] r^{-m-\lambda_{1}-2} \omega(r) \\
& -b_{2}(1+t)^{k}\left[\left(N-1-2\left(m+\lambda_{1}\right)-4 k\right)-C_{\omega}\right] r^{-m-\lambda_{1}-2 k-1}\left|\omega^{\prime}(r)\right| \\
\leq & -k b_{2}(1+t)^{k-1} r^{-m-\lambda_{1}-2 k} \omega(r) \\
& +b_{1}\left[\left(N-2-m-\lambda_{1}\right)\left(m+\lambda_{1}\right)+\tilde{c}\left(N-1-2\left(m+\lambda_{1}\right)\right)\right] r^{-m-\lambda_{1}-2} \omega(r) \\
= & r^{-} m-\lambda_{1}-2 \omega(r)\left(-k b_{2}\left(\frac{r^{2}}{1+t}\right)\right. \\
& \left.+b_{1}\left[\left(N-2-m-\lambda_{1}\right)\left(m+\lambda_{1}\right)+\tilde{c}\left(N-1-2\left(m+\lambda_{1}\right)\right)\right]\right)
\end{aligned}
$$




$$
\begin{aligned}
\leq & r^{-m-\lambda_{1}-2} \omega(r)\left(-k b_{2}\left(\frac{b_{2}}{b_{1}}\right)^{\frac{1-k}{k}}\right. \\
& \left.+b_{1}\left[\left(N-2-m-\lambda_{1}\right)\left(m+\lambda_{1}\right)+\tilde{c}\left(N-1-2\left(m+\lambda_{1}\right)\right)\right]\right)
\end{aligned}
$$

This implies the claim.

Next we use the subsolutions $U_{\text {in }}$ and $U_{\text {out }}$ to prove a lower bound for the solution of $(2.1)$.

Lemma 4.3. Let $N \geq 11, p>p_{c}$ and $U_{0} \in C^{0}([0, \infty))$ such that

$$
U_{0}(r) \geq(1+r)^{-m-\lambda_{1}} \omega(r) \quad \text { for } r \geq 0 .
$$

Then the solution $U$ of (2.1) fulfills

$$
U(0, t) \geq C \omega\left(B(t+1)^{\frac{1}{2}}\right) \quad \text { for all } t \geq 0
$$

with some positive constants $B$ and $C$.

Proof. In order to construct a suitable positive subsolution of (2.1), we first fix some constants. We choose $b_{1}>0$ such that

$$
U_{0}(r) \geq(1+r)^{-m-\lambda_{1}} \omega(r) \geq b_{1} r^{-m-\lambda_{1}} \omega(r) \text { for all } r \geq 1 .
$$

Then we fix $k$ and $b_{2}$ like in Lemma 4.2 such that additionally

$$
b_{2} \geq b_{1}
$$

is valid. Furthermore,

$$
\psi(r) \leq c_{\alpha}^{+} r^{-m-\lambda_{1}} \quad \text { for } r>0
$$

is fulfilled for some positive constant $c_{\alpha}^{+}$by Lemma 2.1. Next, we define the constant

$$
B:=\left(\frac{2 b_{2}}{b_{1}}\right)^{\frac{1}{2 k}}>1
$$

in Lemma 4.1 and fix $c>0$ small such that

$$
\frac{b_{1}}{2}-c c_{\alpha}^{+}>0
$$

is satisfied.

Now let $U_{\text {in }}$ and $U_{\text {out }}$ denote the subsolutions of (2.1) defined in Lemmas 4.1 and 4.2, respectively. Then, following [9], we see that

$$
r^{*}(t):=\sup \left\{r>0 \mid c U_{\text {in }}(\rho, t)>U_{\text {out }}(\rho, t) \text { for all } \rho \in[0, r)\right\}
$$

is well-defined with $r^{*}(t) \in(0, \infty]$ for any $t \geq 0$, since $U_{\text {in }}(r, t)$ is positive and $U_{\text {out }}(r, t)=0$ for small $r \geq 0$. We now show that we have $r^{*}(t) \in(0, \infty)$ since $c>0$ has been chosen small enough. Fixing $t \geq 0$, we define

$$
r_{0}(t):=\left(\frac{2 b_{2}}{b_{1}}\right)^{\frac{1}{2 k}}(1+t)^{\frac{1}{2}}>1
$$


and, due to (4.1)-(4.4), obtain

$$
\begin{aligned}
& U_{\text {out }}\left(r_{0}, t\right)-c U_{\text {in }}\left(r_{0}, t\right) \\
& \quad=b_{1} r_{0}^{-m-\lambda_{1}} \omega\left(r_{0}\right)-b_{2}(1+t)^{k} r_{0}^{-m-\lambda_{1}-2 k} \omega\left(r_{0}\right)-c \omega\left(B(t+1)^{\frac{1}{2}}\right) \psi\left(r_{0}\right) \\
& \quad \geq r_{0}^{-m-\lambda_{1}} \omega\left(r_{0}\right)\left[b_{1}-b_{2}(1+t)^{k} r_{0}^{-2 k}\right]-c c_{\alpha}^{+} r_{0}^{-m-\lambda_{1}} \omega\left(B(t+1)^{\frac{1}{2}}\right) \\
& \quad=r_{0}^{-m-\lambda_{1}} \omega\left(r_{0}\right)\left[\frac{b_{1}}{2}-c c_{\alpha}^{+}\right] \\
& >0 .
\end{aligned}
$$

Thus, we conclude $0<r^{*}(t)<r_{0}(t)<\infty$ for every $t \geq 0$ and define

$$
U^{-}(r, t):= \begin{cases}c U_{\text {in }}(r, t) & \text { for } 0 \leq r<r^{*}(t), \\ U_{\text {out }}(r, t) & \text { for } r \geq r^{*}(t),\end{cases}
$$

and $t \geq 0$. As $U_{r}^{-}(0, t)=0$ by $(2.2), U^{-}$is a subsolution of (2.1) by Lemmas 4.1 and 4.2. As moreover

$$
0<C_{2} U^{-}(r, 0) \leq U_{0}(r) \text { for } r \geq 0
$$

is satisfied for some small constant $C_{2}>0$, the comparison principle implies

$$
0<C_{2} U^{-}(r, t) \leq U(r, t) \text { for } r \geq 0, t \geq 0 .
$$

Hence, the proof is complete since

$$
U^{-}(0, t)=c U_{\text {in }}(0, t)=c \omega\left(B(t+1)^{\frac{1}{2}}\right)
$$

is fulfilled for all $t \geq 0$ by (2.2).

Now we immediately obtain a lower estimate for the evolution of the difference of two solutions to (1.1). This result generalizes Theorem 1.1(b).

Proposition 4.4. Let $N \geq 11$ and $p>p_{c}$. Furthermore, suppose $u_{0}, \tilde{u}_{0} \in$ $C^{0}\left(\mathbb{R}^{N}\right)$ fulfill

$$
\varphi_{\alpha}(|x|) \leq \tilde{u}_{0}(x) \leq u_{0}(x) \leq \varphi_{\infty}(|x|), \quad x \in \mathbb{R}^{N} \backslash\{0\},
$$

and

$$
u_{0}(x)-\tilde{u}_{0}(x) \geq c_{2}(1+|x|)^{-m-\lambda_{1}} \omega(|x|), \quad x \in \mathbb{R}^{N},
$$

with some positive constants $\alpha$ and $c_{2}$. Then there are positive constants $B_{2}$ and $C_{2}$ such that the solutions $u$ and $\tilde{u}$ of (1.1) satisfy

$$
\|u(\cdot, t)-\tilde{u}(\cdot, t)\|_{L^{\infty}\left(\mathbb{R}^{N}\right)} \geq C_{2} \omega\left(B_{2}(t+1)^{\frac{1}{2}}\right) \quad \text { for all } t \geq 0 .
$$

Proof. By choosing

$$
U_{0}(r):=c_{2}(1+r)^{-m-\lambda_{1}} \omega(r), \quad r \geq 0
$$

we obtain

$$
u_{0}(x)-\tilde{u}_{0}(x) \geq U_{0}(|x|)>0, \quad x \in \mathbb{R}^{N} .
$$

Hence, Lemmas 2.2 (ii) and 4.3 imply

$$
\|u(\cdot, t)-\tilde{u}(\cdot, t)\|_{L^{\infty}\left(\mathbb{R}^{N}\right)} \geq U(0, t) \geq C \omega\left(B(t+1)^{\frac{1}{2}}\right) \quad \text { for all } t \geq 0
$$

with some positive constants $B$ and $C$. 
Finally, the second part of Theorem 1.1 is a consequence of Proposition 4.4 .

Proof of Theorem 1.1(b): In case of $\alpha>0$ we choose $u_{0}$ such that (1.10) and

$$
u_{0}(x)-\varphi_{\alpha}(|x|) \geq c_{2}(1+|x|)^{-m-\lambda_{1}} \omega(|x|), \quad x \in \mathbb{R}^{N},
$$

is satisfied. Then Proposition 4.4 implies (1.13).

In case of $\alpha<0$ we fix $u_{0}$ fulfilling (1.10) and

$$
u_{0}(x)-\varphi_{\alpha}(|x|) \leq-c_{2}(1+|x|)^{-m-\lambda_{1}} \omega(|x|), \quad x \in \mathbb{R}^{N} .
$$

Hence, we can apply Proposition 4.4 to the functions $-u$ and $\varphi_{-\alpha}=-\varphi_{\alpha}$ and obtain

$$
\left\|-u(\cdot, t)-\varphi_{-\alpha}(|\cdot|)\right\|_{L^{\infty}\left(\mathbb{R}^{N}\right)} \geq C_{2} \omega\left(B_{2}(t+1)^{\frac{1}{2}}\right) \quad \text { for all } t \geq 0
$$

with some positive constants $B_{2}$ and $C_{2}$. Now this implies (1.13) in view of $\varphi_{-\alpha}=-\varphi_{\alpha}$.

\section{Acknowledgments}

The author would like to thank Marek Fila for tending the author's attention towards this problem.

\section{References}

[1] Berestycki, H., Lions, P.L., Peletier, L.A.: An ODE approach to existence of positive solutions for semilinear problems in $\mathbb{R}^{N}$. Indiana Univ. Math. J. 30, 141-157 (1981)

[2] Chen, W., Li, C.: Qualitative properties of solutions to some nonlinear elliptic equations in $\mathbb{R}^{2}$. Duke Math. J. 71, 427-439 (1993)

[3] Fila, M., King, J.R., Winkler, M., Yanagida, E.: Optimal lower bound of the grow-up rate for a supercritical parabolic equation. J. Differ. Equ. 228, 339-356 (2006)

[4] Fila, M., King, J.R., Winkler, M., Yanagida, E.: Grow-up rate of solutions of a semilinear parabolic equation with a critical exponent. Adv. Differ. Equ. 12, $1-26$ (2007)

[5] Fila, M., King, J.R., Winkler, M., Yanagida, E.: Linear behaviour of solutions of a superlinear heat equation. J. Math. Anal. Appl. 340, 401-409 (2008)

[6] Fila, M., King, J.R., Winkler, M., Yanagida, E.: Very slow grow-up of solutions of a semilinear parabolic equation (submitted)

[7] Fila, M., Winkler, M.: Rate of convergence to a singular steady state of a supercritical parabolic equation. J. Evol. Equ. 8, 673-692 (2008)

[8] Fila, M., Winkler, M., Yanagida, E.: Grow-up rate of solutions for a supercritical semilinear diffusion equation. J. Differ. Equ. 17, 365-389 (2004) 
[9] Fila, M., Winkler, M., Yanagida, E.: Convergence rate for a parabolic equation with supercritical nonlinearity. J. Dyn. Differ. Equ. 17, 249-269 (2005)

[10] Fila, M., Winkler, M., Yanagida, E.: Slow convergence to zero for a parabolic equation with supercritical nonlinearity. Math. Ann. 340, 477-496 (2008)

[11] Fila, M., Winkler, M., Yanagida, E.: Convergence to self-similar solutions for a semilinear parabolic equation. Discret. Contin. Dyn. Syst. 21, 703-716 (2008)

[12] Fila, M., Winkler, M., Yanagida, E.: Convergence of solutions of a semilinear parabolic equation to selfsimilar solutions of the linear heat equation. Adv. Differ. Equ. 13, 1131-1149 (2008)

[13] Fujita, H.: On the blowing up of solutions of the Cauchy problem for $u_{t}=\Delta u+u^{1+\alpha}$. J. Fac. Sci. Univ. Tokyo, Sect. I 13, 109-124 (1966)

[14] Gidas, B., Spruck, J.: Global and local behavior of positive solutions of nonlinear elliptic equations. Commun. Pure Appl. Math. 34, 525-598 (1981)

[15] Gui, C., Ni, W.-M., Wang, X.: On the stability and instability of positive steady states of a semilinear heat equation in $\mathbb{R}^{n}$. Commun. Pure Appl. Math. 45, 1153$1181(1992)$

[16] Gui, C., Ni, W.-M., Wang, X.: Further study on a semilinear heat equation. J. Differ. Equ. 169, 588-613 (2001)

[17] Hoshino, M., Yanagida, E.: Sharp estimates of the convergence rate for a semilinear parabolic equation with supercritical nonlinearity. Nonlinear Anal. TMA 69, 3136-3152 (2008)

[18] Lee, T.-Y., Ni, W.-M.: Global existence, large time behavior and life span of solutions of a semilinear parabolic Cauchy problem. Trans. Am. Math. Soc. 333, 365-378 (1992)

[19] Li, Y.: Asymptotic behavior of positive solutions of equation $\Delta u+K(x) u^{p}=0$ in $\mathbb{R}^{n}$. J. Differ. Equ. 95, 304-330 (1992)

[20] Mizoguchi, N.: Growup of solutions for a semilinear heat equation with supercritical nonlinearity. J. Differ. Equ. 227, 652-669 (2006)

[21] Poláčik, P., Quittner, P., Souplet, P.: Singularity and decay estimates in superlinear problems via Liouville-type theorems. Part II: Parabolic equations. Indiana Univ. Math. J. 56, 879-908 (2007)

[22] Poláčik, P., Yanagida, E.: On bounded and unbounded global solutions of a supercritical semilinear heat equation. Math. Ann. 327, 745-771 (2003)

[23] Poláčik, P., Yanagida, E.: A Liouville property and quasiconvergence for a semilinear heat equation. J. Differ. Equ. 208, 194-214 (2005)

[24] Quittner, P., Souplet, P.: Superlinear Parabolic Problems. Blow-up, Global Existence and Steady States. Birkhäuser Advanced Texts/Basler Lehrbücher, Basel (2007) 
[25] Souplet, P., Weissler, F.: Regular self-similar solutions of the nonlinear heat equation with initial data above the singular steady state. Ann. Inst. Henri Poincaré, Anal. Non Linéaire 20, 213-235 (2003)

[26] Stinner, C.: Very slow convergence to zero for a supercritical semilinear parabolic equation. Adv. Differ. Equ. 14, 1085-1106 (2009)

[27] Wang, X.: On the Cauchy problem for reaction-diffusion equations. Trans. Am. Math. Soc. 337, 549-590 (1993)

C. Stinner

Fachbereich Mathematik,

Universität Duisburg-Essen, 45117 Essen,

Germany.

e-mail: christian.stinner@uni-due.de

Received: 23 June 2009.

Accepted: 16 November 2009. 